\title{
Mastectomia e Sexualidade: Uma Revisão Integrativa
}

\author{
Mastectomy and Sexuality: An Integrative Review
}

\author{
Vanessa Monteiro Cesnik \& Manoel Antônio dos Santos* \\ Universidade de São Paulo, Ribeirão Preto, Brasil
}

\begin{abstract}
Resumo
Mulheres acometidas pelo câncer mamário são confrontadas com a retirada de uma parte do corpo relacionada à sexualidade. Este estudo teve por objetivo investigar o impacto do câncer de mama e da mastectomia na sexualidade da mulher por meio da análise da produção científica publicada no período de 2000 a 2009. O levantamento bibliográfico foi realizado a partir das bases de dados LILACS, MedLine e PsycINFO. Foram selecionados 29 artigos, que constituíram o corpus do estudo. Os achados foram agrupados por meio de análise de conteúdo. Os resultados evidenciaram que, após a retirada da mama, o desempenho sexual é comprometido, com redução da freqüência de intercurso sexual nos primeiros estágios do tratamento e diminuição da excitação, interesse e satisfação sexual.

Palavras-chave: Mastectomia, sexualidade, neoplasias da mama, comportamento sexual, revisão da literatura.
\end{abstract}

\begin{abstract}
Women affected by breast cancer are faced with the removal of a body part related to sexuality. This study aimed to investigate the impact of breast cancer and mastectomy in women's sexuality through the analysis of scientific literature published from 2000 to 2009. The bibliographic survey was carried out in LILACS, MedLine and PsycINFO databases. Twenty nine articles were selected to constitute the corpus of the analysis. The findings were grouped by means of content analysis. The results showed that after the removal of the breast, sexual performance is compromised, with reduced frequency of sexual intercourse in the early stages of treatment and low sexual desire, interest and satisfaction.

Keywords: Mastectomy, sexuality, breast neoplasms, sexual behavior, literature review.
\end{abstract}

O presente estudo propõe-se a explorar as consequências do câncer de mama e da mastectomia sobre a sexualidade da mulher acometida. Para tanto, será abordado, preliminarmente, o conceito de sexualidade e suas possíveis compreensões no campo da saúde, bem como as possíveis interferências da doença e da cirurgia para remoção da massa tumoral na vivência sexual feminina.

A oncologia é um dos contextos de atuação que mais colocam o profissional em contato com questões da sexualidade, especialmente quando o órgão acometido é a mama, no caso das mulheres, ou a próstata, nos pacientes masculinos (Barton-Burke \& Gustason, 2007). O câncer de mama é, provavelmente, o tipo de neoplasia que mais amedronta as mulheres, tanto por sua alta prevalência, como por seus efeitos psicológicos e físicos (Peres \& Santos, 2009). A doença produz alterações importantes

* Este estudo foi financiado pela Fundação de Amparo à Pesquisa do Estado de São Paulo (FAPESP), processo $\mathrm{n}^{\circ}$ 15163-7, mediante bolsa de Iniciação Científica concedida à primeira autora, sob orientação do segundo autor

Endereço para correspondência: Departamento de Psicologia, Faculdade de Filosofia, Ciências e Letras de Ribeirão Preto, Universidade de São Paulo, Av. Bandeirantes, 3900, Monte Alegre, Ribeirão Preto, SP, Brasil 14040-901. E-mail: masantos@ffclrp.usp.br na imagem corporal da mulher, que podem afetar sua vivência da sexualidade e satisfação conjugal (Instituto Nacional do Câncer, 2009; Scorsolini-Comin, Santos, \& Souza, 2009). Apesar de a sobrevivência ser a meta principal do tratamento, não se pode desviar o olhar dos aspectos subjetivos e relacionais, que são profundamente associados ao estado de bem-estar físico, psíquico, social, existencial e espiritual que compõem a qualidade de vida total da paciente (Huguet, Morais, Osis, Pinto-Neto, \& Gurgel, 2009).

A sobrevivência é constituída de diferentes fases, cada uma delas com suas demandas específicas, que afetam a adaptação psicossocial de diferentes maneiras. Entretanto, a maioria dos estudos disponíveis na área da psicooncologia se refere às fases do diagnóstico e do tratamento, havendo poucos trabalhos destinados a investigar a situação psicossocial das pacientes no período pós-tratamento (Silva \& Santos, 2008, 2009, 2010). Questões como o impacto sobre a autoestima da mulher, o relacionamento conjugal e a retomada da vida sexual configuram temas de pesquisa que solicitam maior atenção.

A mastectomia consiste na cirurgia para retirada da massa tumoral. As intervenções cirúrgicas podem variar de acordo com extensão da mama que sofre a mutilação. Existem cirurgias conservadoras, como a nodulectomia e 
a quadrantectomia, e as intervenções radicais, com a total ablação da mama e músculos correlatos. No período imediatamente após a mastectomia há uma retomada progressiva dos hábitos que, anteriormente, organizavam o cotidiano das mulheres. Esses hábitos foram momentaneamente alterados pela doença e seus tratamentos. Porém, é no momento pós-tratamento que aparecem mais claramente as repercussões no plano afetivo-sexual e também as dificuldades de adaptação à nova situação de vida, com limitações físicas e restrições de vários tipos (Duarte \& Andrade, 2003; Rossi \& Santos, 2003).

Algumas mulheres mastectomizadas podem se sentir sexualmente repulsivas, a ponto de chegarem a evitar manter contatos sexuais. Das reclamações mais frequentes observadas destacam-se: o medo de não ser mais atraente sexualmente e a sensação de diminuição da feminilidade (Arán et al., 1994), acarretando prejuízo da autoestima.

De acordo com estudo de revisão (Barton-Burke \& Gustason, 2007) sabe-se, empiricamente, que existem barreiras para as intervenções que abordem a sexualidade de mulheres com câncer. Essas barreiras decorrem dos pressupostos implícitos sobre sexualidade, tanto por parte do paciente quanto do próprio cuidador. $\mathrm{O}$ que ocorre na prática é que o tema da sexualidade acaba por ser marginalizado na assistência e pode não ser discutido com a paciente pelo cuidador, o que sinaliza à doente que ele também não pode levantar o tema em questão, caso sinta necessidade. As autoras mencionam que pacientes relatam desejo de poder discutir questões sobre sexualidade com o profissional de saúde, mas se sentem constrangidas e relutam em fazê-lo.

Cabe ao profissional de saúde tentar clarificar as questões que as mulheres têm nos mais variados atendimentos, já que as dificuldades em viver a sexualidade são mais comuns do que se pode imaginar. Entretanto, a ausência de abertura na assistência impossibilita que se forme um vínculo entre paciente oncológico e profissional, o que torna difícil a verbalização do problema (Gozzo, Fustinoni, Barbieri, Roehr, \& Freitas, 2000).

A literatura evidencia que não apenas a sexualidade, como também a vida conjugal são dimensões ainda negligenciadas nos cuidados em saúde (Barton-Burke \& Gustason, 2007; Talhaferro, Lemos, \& Oliveira, 2007). Para mudar essa situação, é necessário que o profissional de saúde envolvido com a assistência oncológica conheça as necessidades da mulher com câncer de mama. Estudos de revisão sistemática da produção científica podem contribuir para preencher essa lacuna, de modo a direcionar novas pesquisas.

Desse modo, justifica-se a proposta do presente estudo, que teve por objetivo investigar o impacto do câncer de mama e da mastectomia na sexualidade da mulher mastectomizada, por meio de uma revisão integrativa da literatura. Acredita-se que mapear, sintetizar e analisar a produção científica nacional e internacional recente, relativa à interferência da mastectomia (como tratamento do câncer de mama) na sexualidade dessas mulheres, considerando 10 anos de contribuição da literatura (2000-2009), pode proporcionar subsídios para instrumentalizar e qualificar intervenções profissionais direcionadas a amparar o sofrimento emocional suscitado pela perda da mama.

\section{Método}

Trata-se de uma pesquisa retrospectiva, descritiva e documental, que teve como recorte temporal o período entre 2000 e 2009. O estudo de revisão foi orientado pela seguinte questão norteadora: $O$ câncer de mama e a mastectomia, como uma das modalidades de tratamento, interferem na sexualidade da mulher acometida? A coleta dos dados incluiu periódicos indexados nas bases de dados bibliográficos MedLine, LILACS e PsycINFO. Esses periódicos têm caráter multidisciplinar e divulgam o conhecimento científico arbitrado, contribuindo para a construção da complexidade do saber na área de interface do câncer de mama e sexualidade.

Para alcançar o objetivo proposto, foram seguidos os seguintes passos metodológicos, preconizados por Broome (2000) para empreender a revisão da literatura: (a) realização de um levantamento sistematizado das publicações nacionais e internacionais sobre sexualidade e mastectomia; (b) identificação dos autores, tipos de pesquisa, ano de publicação, periódicos nos quais foram veiculados tais estudos, origem dos artigos, idioma em que foram redigidos, objetivos e resultados obtidos; (c) análise descritiva dos resultados dos estudos e avaliação crítica das contribuições oferecidas para a produção de conhecimento sobre a temática.

A revisão integrativa foi escolhida como recurso metodológico porque permite sumarizar estudos realizados anteriormente na temática selecionada, colocando em discussão os métodos, fontes, objetivos e resultados, o que permite estabelecer conclusões em relação ao campo de conhecimento demarcado (Broome, 2000). A coleta de dados foi realizada no período de abril a maio de 2010. Foram pesquisados os artigos indexados com as seguintes palavras-chave: "mastectomy", "breast neoplasms", "sexuality", "sexual behavior", "amputation", "psychossexual development", "marital relations". Estes descritores foram escolhidos de acordo com o DECS Descritores em Ciências da Saúde (as quatro primeiras palavras-chave) e Terminologia Psi (as três últimas mencionadas). A busca foi realizada por meio do sistema SIBI da Universidade de São Paulo, uma rede de serviços que inclui um catálogo on line que franqueia o acesso às bases de dados e conteúdos dos periódicos indexados, possibilitando a obtenção, na íntegra, de artigos publicados em revistas disponibilizadas.

$\mathrm{Na}$ pesquisa bibliográfica foram considerados como critérios de inclusão para busca dos artigos os seguintes parâmetros: (a) artigos circunscritos ao câncer de mama feminino; (b) artigos escritos na língua inglesa, portu- 
Cesnik, V. M. \& Santos, M. A. (2012). Mastectomia e Sexualidade: Uma Revisão Integrativa.

guesa ou espanhola; (c) publicados entre 2000 e 2009; (d) que apresentavam resultados empíricos; (e) que disponibilizavam o resumo nas bases de dados; (f) publicações que abordavam a mastectomia como tratamento para o câncer de mama e suas repercussões sobre a sexualidade das mulheres acometidas; e (g) que tratavam desses assuntos sob a perspectiva da mulher mastectomizada e não segundo a percepção de outras pessoas (familiares, cônjuges, profissionais) em relação a elas.

Como critérios de exclusão, estabeleceram-se os seguintes limites: (a) apresentação sob formato de dissertação, tese, capítulo de livro, livro, manual, editorial, resenha, comentário ou crítica; (b) artigos sobre mastectomia profilática; (c) artigos oriundos de estudos de revisão da literatura; (d) relatos de pesquisa com mulheres que tiveram recorrência do câncer de mama ou metástase; (e) estudos realizados somente com mulheres que fizeram cirurgia de reconstrução da mama.

Após a leitura dos resumos, empreendeu-se a recuperação dos artigos selecionados, de acordo com os critérios de inclusão e exclusão. Esses artigos na íntegra compuseram o corpus da pesquisa. Após a leitura dos artigos procedeu-se a extração dos dados de interesse para a revisão. As informações foram registradas em um formu- lário de identificação, preparado previamente com base na literatura (Broome, 2000). O formulário foi preenchido para cada artigo da amostra, o que permitiu a sistematização dos dados, que posteriormente foram organizados em uma pasta e catalogados em ordem numérica crescente por ano de publicação. Após leituras exaustivas, os achados das publicações foram submetidos à análise de conteúdo (Minayo, 2004). Essa análise desdobrouse em três etapas: (a) pré-análise, (b) exploração do material e (c) tratamento dos resultados obtidos. Os dados foram organizados segundo os conteúdos que emergiram no corpus investigado e analisados por meio de estatística descritiva (porcentagem e distribuição de frequências).

\section{Resultados e Discussão}

A amostra final foi composta por artigos indexados nas três bases de dados escolhidas e que preencheram os critérios de inclusão utilizados para qualificar as publicações no processo de busca bibliográfica. Dos 224 estudos listados, 43 satisfizeram esses critérios. Descartando-se os artigos repetidos em mais de uma base indexadora, 29 foram selecionados conforme os critérios preestabelecidos e, assim, constituíram o corpus deste estudo.

Tabela 1

Distribuição dos Artigos que Constituem o Corpus do Estudo Segundo Autores, Ano de Publicação e Título

\begin{tabular}{|c|c|c|c|}
\hline Ref. & Autor(es) & Ano & Título \\
\hline 1 & $\begin{array}{l}\text { Al-Ghazal, Fallowfield, } \\
\text { \& Blamey }\end{array}$ & 2000 & $\begin{array}{l}\text { Comparison of psychological aspects and patient satisfaction following } \\
\text { breast conserving surgery, simple mastectomy and breast reconstruction }\end{array}$ \\
\hline 2 & Yurek, Farrar, \& Andersen & 2000 & $\begin{array}{l}\text { Breast cancer surgery: Comparing surgical groups and determining } \\
\text { individual differences in postoperative sexuality and body change stress }\end{array}$ \\
\hline 3 & Fung, Lau, Fielding, Or, \& Yip & 2001 & $\begin{array}{l}\text { The impact of mastectomy, breast-conserving treatment and immediate } \\
\text { breast reconstruction on the quality of life of Chinese women }\end{array}$ \\
\hline 4 & Ming & 2002 & Psychological predictors of marital adjustment in breast cancer patients. \\
\hline 5 & Taylor et al. & 2002 & $\begin{array}{l}\text { Treatment regimen, sexual attractiveness concerns and psychological } \\
\text { adjustment among African American breast cancer patients }\end{array}$ \\
\hline 6 & Duarte \& Andrade & 2003 & $\begin{array}{l}\text { Enfrentando a mastectomia: Análise dos relatos de mulheres } \\
\text { mastectomizadas sobre questões ligadas à sexualidade }\end{array}$ \\
\hline 7 & $\begin{array}{l}\text { Ganz, Greendale, Petersen, } \\
\text { Kahn, \& Bower }\end{array}$ & 2003 & $\begin{array}{l}\text { Breast cancer in younger women: Reproductive and late health effects of } \\
\text { treatment }\end{array}$ \\
\hline 8 & Kagawa-Singer \& Wellisch & 2003 & $\begin{array}{l}\text { Breast cancer patients' perceptions of their husbands'support in a cross- } \\
\text { cultural context }\end{array}$ \\
\hline 9 & Avis, Crawford, \& Manuel & 2004 & Psychosocial problems among younger women with breast cancer \\
\hline 10 & Bukovic et al. & 2004 & $\begin{array}{l}\text { Differences in sexual functioning between patients with benign and } \\
\text { malignant breast tumors }\end{array}$ \\
\hline 11 & $\begin{array}{l}\text { Engel, Kerr, Schlesinger-Raab, } \\
\text { Sauer, \& Holzel }\end{array}$ & 2004 & $\begin{array}{l}\text { Quality of life following breast-conserving therapy or mastectomy: Results } \\
\text { of a 5-year prospective study }\end{array}$ \\
\hline 12 & Ganz et al. & 2004 & $\begin{array}{l}\text { Quality of life at the end of primary treatment of breast cancer: First } \\
\text { results from the moving beyond cancer randomized trial }\end{array}$ \\
\hline 13 & Souto \& Souza & 2004 & Sexualidade da mulher após a mastectomia \\
\hline 14 & Yeo et al. & 2004 & $\begin{array}{l}\text { Psychosocial impact of breast cancer surgeries in Chinese patients and } \\
\text { their spouses }\end{array}$ \\
\hline 15 & Conde et al. & 2005 & $\begin{array}{l}\text { Menopause symptoms and quality of life in women aged } 45 \text { to } 65 \text { years } \\
\text { with and without breast cancer }\end{array}$ \\
\hline
\end{tabular}


16 Monteiro-Grillo,

Marques-Vidal, \& Jorge

17 Speer et al.

18 Takahashi \& Kai

19 Wimberly, Carver, Laurenceau, 2005 Harris, \& Antoni

20 Fatone, Moadel, Foley, Fleming, \& Jandorf

21 Madeira, Almeida, \& Jesus

22 Talhaferro et al.

23 Takahashi et al.

24 Abasher

25 Alicikus et al.

26 Gorisek, Krajnc, \& Krajnc

27 Huguet et al.

28 Lam, Chan, Hung, Or, \& Fielding 2009

29 Markopoulos et al.
2005

2005

2005

2007

2007

2007

2008

2009

2009

2009
Psychosocial effect of mastectomy versus conservative surgery in patients with early breast cancer

Study of sexual functioning determinants in breast cancer survivors Sexuality after breast cancer treatment: Changes and coping strategies among Japanese survivors

Perceived partner reactions to diagnosis and treatment of breast cancer: Impact on psychosocial and psychosexual adjustment

Urban voices: The quality-of-life experience among women of color with breast cancer

Refletindo sobre a sexualidade da mulher mastectomizada

Mastectomia e suas consequências na vida da mulher

Impact of breast cancer diagnosis and treatment on women's sexuality:

A survey of Japanese patients

Sexual health issues in Sudanese women before and during hormonal treatment for breast cancer

Psychosexual and body image aspects of quality of life in turkish breast cancer patients: A comparison of breast conserving treatment and mastectomy

Quality of life and the effect on social status among Slovenian women after breast cancer treatment

Qualidade de vida e sexualidade de mulheres tratadas de câncer de mama Social adjustment among Chinese women following breast cancer surgery Impact of breast cancer surgery on the self-esteem and sexual life offemale patients
Do total de artigos encontrados nas diferentes combinações, houve diferença na eficácia das combinações de palavras-chave de acordo com a base de dados pesquisada. Assim, a combinação "breast neoplasms" e "sexuality", bem como a combinação "breast neoplasms" e "sexual behavior" mostraram-se as mais promissoras para a base de dados MedLine, enquanto que a combinação "mastectomy" e "sexuality" mostrou-se mais eficaz na busca empreendida na base de dados LILACS. Já a combinação "breast neoplasms" e "marital relations", assim como "breast neoplasms" e "sexuality", trouxeram mais artigos quando a procura foi realizada na base PsycINFO. Esses resultados podem ser melhor apreciados na Tabela 2 .

No que concerne ao ano de publicação, observou-se que os anos de 2004 e 2009 concentraram o maior número de publicações, com $20,7 \%$ cada, totalizando, juntos, $41,4 \%$ da amostra total de artigos que compõem o corpus do estudo (Tabela 2). Em seguida, despontou o ano de

Tabela 2

Distribuição Numérica de Publicações Encontradas e Selecionadas nas Bases Indexadoras Consultadas

\begin{tabular}{|c|c|c|c|c|c|c|c|c|}
\hline \multicolumn{3}{|c|}{ Palavras-chave } & \multicolumn{2}{|c|}{ MedLine } & \multicolumn{2}{|c|}{ LILACS } & \multicolumn{2}{|c|}{ PsycINFO } \\
\hline & & & Enc. & Selec. & Enc. & Selec. & Enc. & Selec. \\
\hline "Mastectomy" & $e$ & "Sexuality" & 22 & 5 & 5 & 3 & 4 & 1 \\
\hline "Mastectomy" & $e$ & "Sexual behavior" & 15 & 5 & - & - & - & - \\
\hline "Mastectomy" & $e$ & "Psychossexual development" & - & - & - & - & - & - \\
\hline "Mastectomy" & $e$ & "Marital relations" & - & - & - & - & 2 & - \\
\hline "Breast neoplasms" & $e$ & "Sexuality" & 74 & 11 & 4 & 1 & 29 & 1 \\
\hline "Breast neoplasms" & $e$ & "Sexual behavior" & 59 & 14 & 2 & - & 2 & - \\
\hline "Breast neoplasms" & $e$ & "Psychossexual development" & - & - & - & - & - & - \\
\hline "Breast neoplasms" & $e$ & "Marital relations" & - & - & - & - & 19 & 2 \\
\hline "Amputation" & $e$ & "Sexuality" & 2 & - & - & - & 3 & - \\
\hline "Amputation" & $e$ & "Sexual behavior" & 3 & - & 1 & - & - & - \\
\hline "Amputation" & $e$ & "Psychossexual development" & - & - & - & - & - & - \\
\hline \multirow[t]{2}{*}{ "Amputation" } & $e$ & "Marital relations" & - & - & - & - & - & - \\
\hline & & Total & 175 & 35 & 12 & 4 & 59 & 4 \\
\hline
\end{tabular}


2005, com $17,2 \%$ das publicações selecionadas. Com o mesmo número de artigos, os anos de 2003 e 2007 contribuíram com $10,3 \%$ das publicações cada um. Com duas publicações $(6,9 \%)$ figuram os anos de 2000 e 2002 , enquanto que, para os anos de 2001 e 2008, foi obtido apenas um artigo selecionado. Nenhum artigo publicado em 2006 foi selecionado para compor o corpus deste estudo.

Quanto ao idioma no qual os artigos foram publicados, verificou-se que $82,8 \%$ foram redigidos na língua inglesa, $17,2 \%$ na língua portuguesa e nenhum na língua espanhola. Vale ressaltar que nas bases de dados MedLine e PsycINFO a maioria dos periódicos indexados publicam, preferencialmente, no idioma inglês.

Os artigos da amostra foram publicados em 20 revistas científicas distintas, de forma que o periódico PsychoOncology aparece como o que concentra o maior volume de publicações referentes à temática da mastectomia e da sexualidade no período estabelecido para análise, com sete artigos (24,1\%). Pode-se observar que o periódico científico destacado se refere estritamente à área de interface da Psicologia e o câncer, o que acaba favorecendo a publicação de pesquisas concernentes à temática focalizada na presente revisão. Além desse periódico, outros três concentraram dois artigos cada $(6,9 \%)$ do total do corpus de análise: The Breast Journal, Journal of International Medical Research e Journal of Consulting and Clinical Psychology. Mais 16 revistas científicas estão representadas no corpus, veiculando apenas um artigo cada, sendo cinco nacionais: Arquivos de Ciência da Saúde, Escola Anna Nery Revista de Enfermagem, Estudos de Psicologia (Natal), REME Revista Mineira de Enfermagem e Revista Brasileira de Ginecologia e Obstetrícia.

Quando investigadas as áreas focalizadas pelos periódicos que veiculam os artigos selecionados, encontrou-se que quase a metade $(41,4 \%)$ dos estudos é proveniente de revistas multidisciplinares (ou interdisciplinares) que atuam em temas focados no câncer ou, de modo mais amplo, na saúde, entre outros. O caráter multidisciplinar pode ser visto também nos autores dos artigos, já que os estudos foram assinados por profissionais de diferentes áreas da saúde (enfermagem, medicina, psicologia, entre outras).

A segunda área mais frequente dos periódicos selecionados foi a Psicologia (com 34,5\% dos artigos publicados), dentre os quais o maior número é temático e uma minoria generalista. Três dos artigos selecionados foram publicados em revistas específicas de medicina, dois em periódicos de enfermagem, um em revista de antropologia e outro de biomedicina.

Quanto ao país de origem do primeiro autor de cada artigo, $31 \%$ dos autores eram oriundos dos Estados Unidos, $20,7 \%$ do Brasil, $13,8 \%$ da China, $6,9 \%$ do Japão e o restante da amostra foi composta por um estudo de cada país: Alemanha, Croácia, Eslovênia, Grécia, Portugal, Reino Unido, Sudão e Turquia. Uma vez que o país de origem sinaliza o contexto no qual se realizam investigações relacionadas à temática considerada, o predomínio das publicações produzidas nos Estados Unidos pode ter sido influenciado pelo critério da língua inglesa escolhida para a seleção dos artigos, já que as revistas de cada país tendem a publicar na língua materna. De modo análogo, o fato de o Brasil figurar como o segundo país com maior números de artigos também é influenciado pela inclusão da língua portuguesa na busca, já que, além das revistas internacionais que publicam em inglês, obteve-se acesso a revistas nacionais no idioma português. Ainda assim, pode-se perceber o número expressivo de publicações nacionais em contraposição aos países de língua espanhola, também incluída na busca.

A identificação dos temas subjacentes aos artigos que resultaram da pesquisa bibliográfica permite definir os eixos que nortearam o desenvolvimento dos estudos, bem como as preocupações que motivaram a elaboração dos artigos, como pode ser visualizado na Tabela 3 .

A Tabela 3 mostra que o foco principal dos estudos recaiu sobre a imagem corporal, imagem sexual e/ou sentimento de atratividade experimentado pela mulher após a cirurgia de retirada parcial ou total da mama. Esses temas totalizam 55\% da amostra de artigos incluídos no presente estudo, o que mostra o interesse dos pesquisadores na investigação do impacto da retirada da mama sobre a sexualidade. Esses estudos enfatizam que a perda da mama é vivenciada pelas mulheres acometidas pelo câncer de mama como um evento traumático. É como se, junto à mama afetada, também fosse amputada a sexualidade, o desejo, o sentimento de feminilidade e a atratividade, o que aumenta a vulnerabilidade das pacientes, que se apresentam ansiosas, deprimidas, inseguras e com medo de enfrentarem a vida sexual após a cirurgia.

$\mathrm{O}$ segundo tema mais abordado entre os estudos selecionados relaciona-se com o funcionamento sexual das mulheres, que apareceu em 52\% dos artigos. Mais da metade dos artigos selecionados investigaram o funcionamento (ou disfunção, desarranjo) sexual em geral e consideraram diversas dimensões da sexualidade para alcançarem tal objetivo. Essa visão global da sexualidade é importante para dar visibilidade para o tema na área da saúde, todavia, conforme mencionado anteriormente, a sexualidade é um construto abrangente, que pressupõe uma integração de diversas dimensões individuais, sociais e culturais, de tal maneira que focalizar somente o resultado geral não permite identificar as dificuldades e facilidades vivenciadas pela mulher com câncer de mama. Esse reducionismo também não permite transformar o conhecimento obtido em possíveis intervenções dos profissionais de saúde no cuidado dispensado a essas mulheres.

O terceiro tema mais abordado nos estudos foi a maneira como a mulher lida com sua sexualidade após a cirurgia. Os modos de enfrentamento utilizados guardam relação estreita com a qualidade do relacionamento com 
Tabela 3

Distribuição Numérica e Frequencial dos Temas que Apareceram como Resultado da Investigação nos Artigos da Amostra

Assuntos / Temas abordados nos resultados

Atratividade / Imagem sexual / Imagem corporal

Funcionamento / Atividade sexual / Disfunção sexual /

Desarranjo sexual (geral)

Relação com o parceiro / "Ser vista" e

"Ser procurada" por ele / Não ter parceiro

Excitação / Desejo / Interesse

Frequência das relações sexuais

Desconforto físico / Dor / Fadiga /

Falta de lubrificação vaginal / Cansaço / Medo /

Insegurança / Depressão / Estresse psicológico

Satisfação sexual / Orgasmo

Dimensões afetivas da sexualidade

Relevância da vida sexual antes da doença

Sexualidade como área de preocupação / Necessidade de receber orientação de profissional da saúde

Novas possibilidades / Melhora na vivência da sexualidade

Qualidade da primeira experiência sexual após a cirurgia
Artigos

$1,2,4,5,6,10,11,14,18,19,20,25,26,27,28,29$

$2,4,7,9,10,11,12,13,16,17,18,22,24,25,29$

$17,18,21,22,29,32,33,34,35,36,39,41$

$2,4,9,12,17,18,20,24,25,26,29$

$2,3,11,12,15,18,19,20,23,26$

$4,9,12,17,18,20,22,25,26$

$9,11,13,17,18,21,28$

$2,12,17,24,25,26$

$6,13,19,20,23,27$

$16,18,22,23,25$

$10,23,25,26$

6,9

19 o parceiro sexual e com o modo como ela é vista por ele, o que compreende $41 \%$ da amostra de publicações. Um dos artigos (Madeira et al., 2007) mostrou que ausência de parceiro sexual fixo pode ser indicativo de maior bemestar para a mulher, devido ao fato de algumas entrevistadas que não tinham um relacionamento estável relatarem que se sentiam mais livres e menos incomodadas no tocante à vivência da sexualidade. Os autores do estudo propõem que esse sentimento de bem-estar pode estar relacionado à ausência de "cobrança", ou seja, de possíveis conflitos que emergem quando se tem um parceiro fixo. Ficou evidenciado nesses estudos que a maneira de lidar com os problemas recorrentes da doença foi atrelada à forma como as mulheres percebiam seu vínculo com o parceiro sexual. Mulheres que percebiam a relação com o parceiro como segura e afetuosa apresentaram menos problemas de lidar com a sexualidade do que aquelas que relataram sentir seu relacionamento amoroso fragilizado.

Abrangendo 38\% do corpus investigado situam-se os artigos que abordam questões relacionadas à excitação, desejo e interesse da mulher pelo sexo. A maioria dos estudos constatou que há um decréscimo do desejo e interesse sexual durante o tratamento para o câncer de mama e que isso ocorre em maior escala em mulheres submetidas à quimioterapia. Este efeito adverso do tratamento, contudo, tende a diminuir ao longo do tempo. Avis et al.
(2004) mostraram, em seu estudo, que maior tempo de diagnóstico (e, provavelmente, maior tempo pós-tratamento) foi indicativo de menos problemas com relação ao interesse sexual. Este achado também foi considerado por Bukovic et al. (2004) que, durante a seleção das participantes da pesquisa, excluíram mulheres com mais de dois anos de cirurgia porque o impacto psicológico da mastectomia diminui com o tempo.

As questões relacionadas à frequência de relações sexuais das mulheres mastectomizadas são tematizadas por $34 \%$ dos artigos recuperados. Nesse conjunto de publicações foram incluídos tanto estudos de comparação entre mulheres com câncer de mama submetidas a diferentes tipos de tratamento como mulheres não acometidas pela doença (Conde et al., 2005; Fung et al., 2001; Ganz et al., 2004; Wimberly et al., 2005; Yurek et al., 2000), bem como pesquisas de comparação entre a frequência das relações antes e depois da cirurgia (Engel et al., 2004; Fatone et al., 2007; Takahashi \& Kai, 2005; Takahashi et al., 2008). Em geral, houve diminuição da frequência de relações sexuais após o câncer de mama, em decorrência de diversos fatores: diminuição do desejo sexual, cansaço, mal-estar relacionado à quimioterapia, sentimento de baixa atratividade, entre outros. Mulheres submetidas às cirurgias mais conservadoras tenderam a apresentar menos mudança da frequência de relações sexuais do 
que aquelas submetidas a procedimentos cirúrgicos mais extensos e invasivos.

Em seguida, perfazendo 31\% dos estudos, aparecem os temas relacionados aos desconfortos físicos (falta de lubrificação vaginal, dor, fadiga, ondas de calor) decorrentes dos tratamentos para o câncer de mama e da menopausa precoce induzida pela terapêutica instituída. Os desconfortos físicos foram, geralmente, mencionados como justificativas para diminuição do desejo e da frequência das relações sexuais (Fatone et al., 2007; Ganz et al., 2004; Ming, 2002; Speer et al., 2005; Takahashi \& Kai, 2005; Talhaferro et al., 2007). A interferência da mastectomia sobre a sexualidade feminina ficou evidenciada pela estreita relação entre desconfortos físicos e dificuldades de retomada das atividades sexuais após a cirurgia de retirada da mama. Esses achados estão em consonância com a literatura, que mostra elevada suscetibilidade da mulher com câncer de mama aos estressores físicos (Silva \& Santos, 2010).

Em 24\% dos artigos foram destacados sentimentos de medo e insegurança, além da depressão e estresse psicológico, como intercorrências vinculadas à vivência da sexualidade após a cirurgia. Os achados que apontam para a vulnerabilidade à depressão e estresse psicológico indicam necessidade de inclusão do acompanhamento psicológico na rotina de cuidado para mulheres em reabilitação, a fim de ajudá-las a elaborar sentimentos e vivências dolorosas. Segundo um dos estudos (Gorisek et al., 2009), depois que as pacientes completam o tratamento cirúrgico do câncer de mama, mais tempo deve ser dedicado a prover aconselhamento sobre o impacto que ele pode ter sobre sua vida sexual, bem como apoio psicológico para ajudá-las a entrar em acordo com as mudanças que experimentaram em seu corpo e aparência pessoal. A vulne-rabilidade à depressão e ao estresse é um dado bem documentado no campo do câncer em geral (Borges et al., 2006; Schmidt, Gabarra, \& Gonçalves, 2011) e do câncer de mama em específico (Silva \& Santos, 2008, 2009, 2010).

De acordo com Venâncio (2004) e Ambrósio e Santos (2011), muitas pesquisas ressaltam a importância da atuação do psicólogo no suporte à paciente e aos familiares após a descoberta do câncer de mama, principalmente devido à desestabilização que esse diagnóstico e seu consequente tratamento podem acarretar na vida da mulher. Esse profissional vai ajudar a mulher a manter seu bem-estar psicológico durante o tratamento. Esses estudos referentes ao câncer de mama comprovam que pacientes que participam de atendimento psicológico apresentam melhor ajustamento à doença, redução dos transtornos mentais, como ansiedade e depressão, melhor adesão ao tratamento e diminuição dos sintomas adversos associados ao câncer e aos tratamentos, podendo até obter um aumento no tempo de sobrevida.

A satisfação sexual elou capacidade de alcançar o orgasmo após a cirurgia são temas que têm sido pouco abordados, a julgar pelos relatos de pesquisas selecionados, tendo aparecido em seis (21\%) dos 29 artigos da amostra. Mesmo considerando que existem outras formas de abordar a satisfação sexual, que podem não ter sido capturadas na presente revisão, esse achado sugere que poucos estudos têm se interessado por essa dimensão da sexualidade.

O número reduzido de estudos dedicados a investigar a satisfação sexual e a capacidade orgástica da mulher mastectomizada sugere que ainda predomina um olhar reducionista (Berman \& Berman, 2003). Para estas autoras fica subentendida, na maior parte dos estudos, a ideia de que, quando a mulher é capaz de manter relações sexuais sem dor, é porque não há nenhum tipo de problema com ela. Além disso, o fato de a educação sexual ter sido excluída das universidades potencializou o problema, já que esse treinamento não faz parte da educação dos profissionais da área de saúde.

Apenas cinco artigos (17\%) abordaram as dimensões afetivas da sexualidade, consideradas, na maioria das vezes, como fatores facilitadores na vivência da sexualidade após o câncer de mama. Fatone et al. (2007) sustentam que o afeto e a intimidade são dimensões que sofreram alterações após a cirurgia, porém outros autores (Duarte \& Andrade, 2003; Huguet et al., 2009; Souto \& Souza, 2004; Wimberly et al., 2005) constataram que a vivência das dimensões afetivas da sexualidade (formas de sedução, troca de carícias, cumplicidade, toque, entre outras) auxiliou na experiência da sexualidade após a cirurgia. Os estudos revisados apresentaram resultados convergentes, que apontam para a importância que o relacionamento afetivo-sexual assume para o processo de recuperação da mulher no período pós-mastectomia. Conforme indicam os achados levantados, há por parte das mulheres valorização dos aspectos afetivos da experiência, que envolvem o relacionamento sexual e expectativa de que o parceiro tome iniciativa no intercurso sexual.

A importância da vida sexual para a mulher e para seu parceiro antes da cirurgia foi levantada como fator relevante para a investigação da vivência da sexualidade após a intervenção cirúrgica em cinco artigos (17\%). Em uma das investigações (Kagawa-Singer \& Wellisch, 2003) foi considerada uma limitação do estudo o fato de não ter sido considerada a história sexual antes do diagnóstico de câncer de mama ou da intervenção cirúrgica, já que está demonstrado que o funcionamento sexual pobre anterior à doença pode ser ainda mais agravado pela cirurgia de câncer de mama. Em concordância com esse fator, outro estudo (Alicikus et al., 2009) estabeleceu que 44\% das pacientes com problemas na vida sexual já os manifestavam antes da cirurgia.

A importância de se proporcionar melhor orientação a respeito das consequencias potenciais dos tratamentos para o cancer de mama sobre a sexualidade da mulher e o reconhecimento de que essa área é motivo de preocupação das pacientes apareceram, de forma destacada, em 
quatro artigos (14\%). Outros artigos enfatizaram ainda a necessidade de produzir um maior número de investigações na área como meio de melhorar a formação dos profissionais de saúde e, por consequência, aprimorar a qualidade dos cuidados prestados às mulheres acometidas pelo câncer de mama (Takahashi \& Kai, 2005). Apesar de muitos estudos postularem como uma das justificativas da pesquisa a necessidade de contribuir para o aprimoramento da formação e capacitação dos profissionais de saúde, muitas vezes o uso das evidências científicas obtidas permanece restrito ao campo científico. De acordo com Costa (2001), é essencial aproximar a produção de conhecimento na área e a prestação de serviços profissionais especializados em psico-oncologia, de modo a elevar o nível técnico das intervenções psicológicas e dos outros profissionais de saúde, exigindo-se a adequação teórico-metodológica dos profissionais à demanda social que se apresenta.

A melhora da vida sexual e da percepção dessa dimensão da vida como campo fértil de novas possibilidades apareceram em dois (7\%) artigos selecionados. Para algumas entrevistadas, as mudanças provocadas pela doença foram positivas, pois fizeram com que valorizassem ainda mais suas vidas e potencializassem novos modos de expressar a própria sexualidade (Duarte \& Andrade, 2003). Apesar dos problemas vivenciados com a retirada da mama, algumas mulheres relataram que sua vida sexual havia melhorado (Avis et al., 2004). Estas vivências particulares evidenciam a necessidade de produzir novos estudos qualitativos que busquem conhecer as singularidades das experiências vividas pelas mulheres, evitando manter somente as generalizações que decorrem de estudos quantitativos, que por sua vez também devem ser estimulados devido ao alto nível de abrangência e síntese proporcionado por esse tipo de pesquisa. Apesar de, na maioria dos estudos, ser relatado uma piora na vida sexual da mulher após o câncer de mama, há casos que divergem deste padrão de experiência, de modo que o profissional de saúde deve estar preparado também para manejar essa possibilidade.

Apenas um artigo considerou a qualidade da primeira experiência sexual após a cirurgia como importante dimensão na vivência da sexualidade da mulher mastectomizada. Considerando que o tempo todo ocorre a ressignificação do papel da sexualidade na vida das pessoas, e que isso acontece em maior escala diante dos acontecimentos críticos da vida, como o diagnóstico de câncer, a qualidade da primeira experiência sexual pósmastectomia possivelmente está relacionada com a maneira como a sexualidade é ressignificada na vida da mulher. Desse modo, a investigação dessa dimensão poderia fornecer informações valiosas para a compreensão do ajustamento afetivo-sexual do casal após o tratamento do câncer de mama.

A identificação do modo como a sexualidade foi abordada nos artigos revisados mostrou que a temática emerge como parte integrante dos domínios que são, habitualmente, considerados na estimativa da qualidade de vida e dos aspectos psicossociais das pessoas. Por conseguinte, colocar o foco do estudo diretamente em questões relativas à sexualidade, como ocorreu em 12 (41\%) dos 29 artigos $(2,6,10,13,17,18,21,22,23,24$, $25,29)$, não é a única maneira de investigar a vida sexual da mulher. Em 10 (35\%) dos artigos selecionados (3, 5, $7,8,11,12,15,20,26,27)$ a investigação se deu por meio da aplicação de questionários que mensuraram a qualidade de vida como um todo, dos quais foram extraídos e destacados os resultados relativos à sexualidade.

Em cinco $(17 \%)$ artigos $(1,9,14,16,28)$ a sexualidade foi compreendida como parte do ajustamento frente ao impacto psicossocial da doença e da retirada da mama na vida da mulher mastectomizada. Em dois (7\%) estudos $(4,19)$ a vida sexual da mulher foi totalmente vinculada à relação marital/conjugal, de modo a serem excluídas as mulheres sem parceiros fixos no critério de seleção das participantes. A adoção desse critério impede a compreensão mais ampla da situação investigada e limita o alcance dos resultados obtidos apenas às mulheres que estão engajadas em relações afetivas estáveis.

Os estudos revisados abarcaram amplo leque de dimensões da sexualidade que se mostraram diretamente afetadas pelos tratamentos do câncer de mama. A interferência da mastectomia sobre a sexualidade feminina ficou evidenciada pelo impacto exercido sobre o sentimento de atratividade sexual, que coloca em questão a relação com o novo corpo em função da perda da mama e seus reflexos sobre a qualidade do vínculo com o parceiro sexual. Outra dimensão relevante que despontou nos estudos foram os múltiplos desconfortos físicos acarretados pelo tratamento. Uma área que se mostrou especialmente comprometida foi do desempenho sexual, com redução da frequência de intercurso sexual nos primeiros estágios do tratamento e diminuição da excitação, interesse e satisfação sexual da mulher.

Dentre os fatores que facilitaram a retomada da vida sexual pós-mastectomia destacaram-se a postura compreensiva e acolhedora do companheiro e sua manifestação de interesse e iniciativa no envolvimento sexual. A dimensão afetiva da vida sexual e a comunicação entre o casal foram valoradas positivamente pelas mulheres, o que denota a importância atribuída à troca de carícias e ao clima de cumplicidade afetiva, que vai além da expressão genital da resposta sexual. Esses achados vão ao encontro de um estudo (Gozzo et al., 2000) que desvelou a falta de diálogo entre o casal como agravante das dificuldades vivenciadas pelas mulheres.

Finalmente, os estudos ressaltaram a importância do papel dos profissionais de saúde envolvidos na reabilitação psicossocial da mulher mastectomizada, principalmente na oferta de orientação preventiva acerca das consequências potencialmente deletérias do tratamento sobre sua qualidade de vida de um modo geral. 
Cesnik, V. M. \& Santos, M. A. (2012). Mastectomia e Sexualidade: Uma Revisão Integrativa.

\section{Considerações Finais}

Os resultados destacados possibilitaram lançar um olhar crítico e reflexivo sobre o acervo de conhecimentos produzidos recentemente sobre o impacto do câncer de mama e da mastectomia em relação à sexualidade da mulher mastectomizada, permitindo apontar limitações e potencialidades que têm implicações para a prática do cuidado na área oncológica.

Uma limitação, mas que não é propriamente desta revisão e sim dos artigos nela incluídos, é que alguns resultados podem mascarar a experiência individual daquelas mulheres com câncer de mama que tiveram a vivência de maior sofrimento emocional do que a média das pacientes e que, por essa razão, podem ter tido mais dificuldades frente ao diagnóstico e tratamento do que outras mulheres com a mesma doença. Outro fator que pode ter contribuído para limitar o alcance dos resultados é que as mulheres que compuseram a casuística dos estudos podem fazer parte da população de pacientes que se adaptaram bem à cirurgia, enquanto que as que foram afetadas emocionalmente tenderiam a recusar a participar das pesquisas. Tais pacientes podem ter dificuldade em responder ou mesmo se recusarem a completar as questões relacionadas a temas como atração sexual, que constam dos questionários.

Esses achados mostram a importância de que, em estudos futuros, sejam levadas em consideração as vivências da sexualidade ao se analisar o processo de adaptação e ajustamento psicossocial das mulheres com câncer de mama. Também são necessários mais estudos de cunho qualitativo, que busquem individualizar as experiências das mulheres, de modo a oferecer contribuições para que o cuidado possa ser integral.

Como o cerne da preocupação deste estudo recai na possibilidade de aprimorar o cuidado às mulheres acometidas pelo câncer de mama, especialmente na esfera da sexualidade, é muito importante que novas investigações originais sejam realizadas para que uma compreensão mais ampla do fenômeno produza melhor integração das ações de cuidado. Além disso, vale ressaltar que, de acordo com os achados, quanto maior o tempo de cirurgia, menos problemas com interesse sexual são encontrados, o que revela a necessidade de estudos que investiguem também mulheres com pouco tempo de diagnóstico e/ou cirurgia até o final do tratamento primário do câncer de mama (Avis et al., 2004).

Finalmente, recomenda-se a realização de outros estudos de revisão, com o propósito de investigar também a visão do marido e/ou parceiro sexual da mulher após a mastectomia. Esta visão pode ser útil para complementar os resultados obtidos pelos estudos que focalizaram a problemática do ponto de vista das mulheres. Essa recomendação parte da premissa de que, para assegurar um cuidado integral à mulher em relação à sua sexualidade, é interessante ajudar os profissionais a perceberem o par- ceiro como possível fator de ajuda na reabilitação psicossocial.

Estudos de revisão sistemática da literatura são relevantes para respaldar práticas e ações de saúde baseadas em evidências científicas. O consumo de literatura científica atualizada é um recurso relevante para fomentar a qualificação dos profissionais de saúde. Atualmente, a assistência oncológica é fundamentada nos princípios da multidisciplinaridade e vem incorporando, paulatinamente, outros profissionais de saúde, como psicólogo, enfermeiro, assistente social, fisioterapeuta e terapeuta ocupacional. Aprender a trabalhar em equipe adquire uma importância crescente na área da oncologia, o que aumenta a predisposição para investir no aperfeiçoamento de estratégias de cuidado em saúde que incluam o acolhimento de aspectos que transcendem as dimensões estritamente físicas.

De modo geral, os resultados deste estudo de revisão proporcionaram subsídios que podem instrumentalizar a formação e aperfeiçoamento dos profissionais que assistem a mulher com câncer de mama, evidenciando a necessidade de promover espaços de acolhimento e escuta ao sofrimento emocional suscitado pelo acometimento por uma doença grave e estigmatizadora. Oferecer escuta às dimensões subjetivas, tais como a vivência da sexualidade após a mastectomia e a qualidade do relacionamento afetivo-sexual da mulher durante e após o tratamento para o câncer de mama, são demandas urgentes para que se possa obter uma otimização dos recursos empregados no processo de reabilitação psicossocial. Além de contribuir para qualificar a prática assistencial, outro passo fundamental é incorporar o conhecimento produzido pelos estudos recentes em políticas públicas de saúde. Este talvez seja o maior desafio a ser enfrentado para a expansão da prática atual em saúde. Para participar desse desafio, o foco na qualidade da assistência é condição necessária para a inovação e adoção de práticas de cuidado inclusivas, humanizadas e baseadas em evidências científicas.

\section{Referências}

Abasher, S. M. (2009). Sexual health issues in Sudanese women before and during hormonal treatment for breast cancer. Psycho-Oncology, 18(8), 858-865.

Al-Ghazal, S. K., Fallowfield, L., \& Blamey, R. W. (2000). Comparison of psychological aspects and patient satisfaction following breast conserving surgery, simple mastectomy and breast reconstruction. European Journal of Cancer, 36(15), 1938-1943.

Alicikus, Z. A., Gorken, I. B., Sen, R. C., Kentli, S., Kinay, M., Alanyali, H., et al. (2009). Psychosexual and body image aspects of quality of life in Turkish breast cancer patients: A comparison of breast conserving treatment and mastectomy. Tumori, 95(2), 212-218.

Ambrósio, D. C. M. \& Santos, M. A. (2011). Vivências de familiares de mulheres com câncer de mama: Uma compreensão fenomenológica. Psicologia: Teoria e Pesquisa, 27(4), $17-26$ 
Arán, M. R., Zahar, S., Delgado, P. G. G., Souza, C. M., Cabral, C. P. S., \& Viegas, M. (1994). Representações de pacientes mastectomizadas sobre doença e mutilação e seu impacto no diagnóstico precoce do câncer de mama. Jornal Brasileiro de Psiquiatria, 45(11), 633-639.

Avis, N. E., Crawford, S., \& Manuel, J. (2004). Psychosocial problems among younger women with breast cancer. PsychoOncology, 13(5), 295-308.

Barton-Burke, M., \& Gustason, C. J. (2007). Sexuality in women with cancer. Nursing Clinics of North America, 42(4), 531554.

Berman, J., \& Berman, L. (2003). Só para mulheres. Rio de Janeiro, RJ: Record.

Borges, A. D. V. S., Silva, E. F., Mazer, S. M., Toniollo, P. B., Valle, E. R. M., \& Santos, M. A. (2006). Percepção da morte pelo paciente oncológico ao longo do desenvolvimento. Psicologia em Estudo (Maringá), 11(2), 361-369.

Broome, M. E. (2000). Integrative literature reviews in the development of concepts. In B. L. Rodgers \& K. A. Knafl (Eds.), Concept development in nursing: Foundations, techniques and applications (2nd $\mathrm{ed}$., pp. 231-250). Philadelphia, PA: W. B. Saunders.

Bukovic, D., Fajdic, J., Strinic, T., Habek, M., Hojsak, I., \& Radakovic, N. (2004). Differences in sexual functioning between patients with benign and malignant breast tumors. Collegium Antropologicum, 28(2), 191-201.

Conde, D. M., Pinto-Neto, A. M., Cabello, C., Sá, D. S., CostaPaiva, L., \& Martinez, E. Z. (2005). Menopause symptoms and quality of life in women aged 45 to 65 years with and without breast cancer. Menopause: The Journal of the North American Menopause Society, 12(4), 436-443.

Costa, A. L., Jr. (2001). O desenvolvimento da psico-oncologia: Implicações para a pesquisa e intervenção profissional em saúde. Psicologia: Ciência e Profissão, 21(2), 36-43.

Duarte, T. P., \& Andrade, A. N. (2003). Enfrentando a mastectomia: Análise dos relatos de mulheres mastectomizadas sobre questões ligadas à sexualidade. Estudos de Psicologia (Natal), 8(1), 155-163.

Engel, J., Kerr, J., Schlesinger-Raab, A., Sauer, H., \& Holzel, D. (2004). Quality of life following breast-conserving therapy or mastectomy: Results of a 5-year prospective study. The Breast Journal, 10(3), 2230-2231.

Fatone, A. M., Moadel, A. B., Foley, F. W., Fleming, M., \& Jandorf, L. (2007). Urban voices: The quality-of-life experience among women of color with breast cancer. Palliative and Supportive Care, 5(2), 115-125.

Fung, K. W., Lau, Y., Fielding, R., Or, A., \& Yip, A. W. C. (2001). The impact of mastectomy, breast-conserving treatment and immediate breast reconstruction on the quality of life of Chinese women. ANZ Journal of Surgery, 71(4), 202-206.

Ganz, P. A., Greendale, G. A., Petersen, L., Kahn, B., \& Bower, J. E. (2003). Breast cancer in younger women: Reproductive and late health effects of treatment. Journal of Clinical Oncology, 21(22), 4184-4193.

Ganz, P. A., Kwan, L., Stanton, A. L., Krupnick, J. L., Rowland, J. H., Meyerowitz, B. E., et al. (2004). Quality of life at the end of primary treatment of breast cancer: First results from the moving beyond cancer randomized trial. Journal of the National Cancer Institute, 96(5), 376-387.
Gorisek, B., Krajnc, P., \& Krajnc, I. (2009). Quality of life and the effect on social status among Slovenian women after breast cancer treatment. Journal of International Medical Research, 37(2), 557-566.

Gozzo, T. O., Fustinoni, S. M., Barbieri, M., Roehr, W. M., \& Freitas, I. A. (2000). Sexualidade feminina: Compreendendo seu significado. Revista Latino-Americana de Enfermagem, 8(3), 84-90.

Huguet, P. R., Morais, S. S., Osis, M. J., Pinto-Neto, A. M., \& Gurgel, M. S. (2009). Qualidade de vida e sexualidade de mulheres tratadas de câncer de mama. Revista Brasileira de Ginecologia e Obstetrícia, 31(2), 61-67.

Instituto Nacional do Câncer. (2009). Estimativas 2010: Incidência de câncer no Brasil. Retrieved December 23, 2009 from http://www.inca.gov.br

Kagawa-Singer, M., \& Wellisch, D. K. (2003). Breast cancer patients' perceptions of their husbands' support in a crosscultural context. Psycho-Oncology, 12(1), 24-37.

Lam, W. W., Chan, M., Hung, W. K., Or, A., \& Fielding, R. (2009). Social adjustment among Chinese women following breast cancer surgery. Psycho-Oncology, 18(11), 1189-1198.

Madeira, A. M. F., Almeida, G. B. S., \& Jesus, M. C. P. (2007). Refletindo sobre a sexualidade da mulher mastectomizada. REME: Revista Mineira de Enfermagem, 11(3), 254-257.

Markopoulos, C., Tsaroucha, A. K., Kouskos, E., Mantas, D., Antonopoulou, Z., \& Karvelis, S. (2009). Impact of breast cancer surgery on the self-esteem and sexual life of female patients. Journal of International Medical Research, 37(1), 182-188.

Minayo, M. C. de S. (2004). O desafio do conhecimento: Pesquisa qualitativa em saúde (8. ed.). São Paulo, SP: Hucitec.

Ming, V. M. W. (2002). Psychological predictors of marital adjustment in breast cancer patients. Psychology, Health \& Medicine, 7(1), 37-51.

Monteiro-Grillo, I., Marques-Vidal, P., \& Jorge, M. (2005). Psychosocial effect of mastectomy versus conservative surgery in patients with early breast cancer. Clinical \& Translational Oncology, 7(11), 499-503.

Peres, R. S. \& Santos, M. A. (2009). Personalidade e câncer de mama: Produção científica em Psico-Oncologia. Psicologia: Teoria e Pesquisa, 25(4), 611-620. Retirado em 12/05/2010, de http://www.revistaptp.unb.br/index.php/ptp/article/view/ 244/8

Rossi, L., \& Santos, M. A. (2003). Repercussões psicológicas do adoecimento e tratamento em mulheres acometidas pelo câncer de mama. Psicologia: Ciência e Profissão, 23(4), 32-41.

Schmidt, B.; Gabarra, L. M., \& Gonçalves, J. R. (2011). Intervenção psicológica em terminalidade e morte: Relato de experiência. Paidéia (Ribeirão Preto), 21(50), 423-430.

Scorsolini-Comin, F., Santos, M. A., \& Souza, L. V. (2009, Janeiro-Abril). Vivências e discursos de mulheres mastectomizadas: Negociações e desafios do câncer de mama. Estudos de Psicologia (Natal), 14(1), 2009, 41-50. Retirado em 12/08/2010, de http://www.scielo.br/pdf/epsic/v14n1/ a06v14n1.pdf.

Silva, G., \& Santos, M. A. (2008). "Será que não vai acabar nunca?": Perscrutando o universo do pós-tratamento do câncer de mama. Texto \& Contexto Enfermagem, 17(3), 561-568. 
Silva, G., \& Santos, M. A. (2009). O universo do pós-tratamento do câncer de mama. In S. R. Pasian, G. Romanelli, \& M. V. Cunha (Eds.), Investigação científica em Psicologia: Aplicações atuais em saúde (pp. 183-206). São Paulo, SP: Vetor.

Silva, G., \& Santos, M. A. (2010). Stressors in breast cancer post-treatment: A qualitative approach. Revista Latino-americana de Enfermagem, 18(4), 688-695.

Souto, M. D., \& Souza, I. E. O. (2004). Sexualidade da mulher após a mastectomia. Revista de Enfermagem Escola Anna Nery, 8(3), 402-410.

Speer, J. J., Hillenberg, B., Sugrue, D. P., Blacker, C., Kresge, C. L., Decker, V. B., et al. (2005). Study of sexual functioning determinants in breast cancer survivors. The Breast Journal, 11(6), 440-447.

Takahashi, M., \& Kai, I. (2005). Sexuality after breast cancer treatment: Changes and coping strategies among Japanese survivors. Social Science \& Medicine, 61(6), 1278-1290.

Takahashi, M., Ohno, S., Inoue, H., Kataoka, A., Yamaguchi, H., Uchida, Y., et al. (2008). Impact of breast cancer diagnosis and treatment on women's sexuality: A survey of Japanese patients. Psycho-Oncology, 17(9), 901-907.

Talhaferro, B., Lemos, S. S., \& Oliveira, E. (2007). Mastectomia e suas conseqüências na vida da mulher. Arquivos de Ciências da Saúde, 14(1), 17-22.

Taylor, K. L., Lamdan, R. M., Siegel, J. E., Shelby, R., Hrywna, M., \& Moran-Klimi, K. (2002). Treatment regimen, sexual attractiveness concerns and psychological adjustment among African American breast cancer patients. Psycho-Oncology, $11(6), 505-517$.

Venâncio, J. L. (2004). Importância da atuação do psicólogo no tratamento de mulheres com câncer de mama. Revista Brasileira de Cancerologia, 50(1), 55-63.

Wimberly, S. R., Carver, C. S., Laurenceau, J-P, Harris, S. D., $\&$ Antoni, M. H. (2005). Perceived partner reactions to diagnosis and treatment of breast cancer: Impact on psychosocial and psychosexual adjustment. Journal of Consulting and Clinical Psychology, 73(2), 300-311.

Yeo, W., Kwan, W. H., Teo, P. M. L., Nip, S., Wong, E., Hin, L. Y., et al. (2004). Psychosocial impact of breast cancer surgeries in Chinese patients and their spouses. PsychoOncology, 13(2), 132-139.

Yurek, D., Farrar, W., \& Andersen, B. L. (2000). Breast cancer surgery: Comparing surgical groups and determining individual differences in postoperative sexuality and body change stress. Journal of Consulting and Clinical Psychology, 68(4), 697-709. 